\title{
Impedance Characteristics of Mimic Human Tooth Root Canal and Its Equivalent Circuit Model
}

\author{
Jui-Hsiung Huang, ${ }^{a}$ Shi-Chern Yen, ${ }^{\mathrm{a}, \mathrm{z}}$ and Chun-Pin $\operatorname{Lin}^{\mathrm{b}, \mathrm{z}}$ \\ ${ }^{a}$ Department of Chemical Engineering, National Taiwan University, Taipei 10617, Taiwan \\ ${ }^{b}$ School of Dentistry and Graduate Institute of Clinical Dentistry, National Taiwan University and National \\ Taiwan University Hospital, Taipei 10051, Taiwan
}

\begin{abstract}
This investigation examined the complicated impedance characteristics of the human tooth root canal using a mimic system that comprised a cylindrical tubule and 5\% agar-gel. The in vitro impedance measurements of root canals were made using a frequency response analyzer and a tubule filled with distilled water or normal saline. An equivalent circuit model of this bioelectric system was proposed; it closely corresponds to the experimental results. On the basis of the proposed equivalent circuit model, the experimental results can be explained reasonably and the effect of the filling of the canal with the electrolytes can be clearly understood. The modified impedance quotient $(Q)$ and the variation ratio of the impedance quotient $\left(S_{Q}\right)$ are taken as the useful indices of the apex. Different impedance behaviors have been identified from $Q$ values in under-apex and over-apex regions, respectively. The $S_{Q}$ was $<0.15$ at the apex location as well as in the overapex region for all electrolytes and could be used to eliminate the effect of the electrolytic conductivity. This study elucidates the complicated electric mechanisms of electrolytic systems and improves clinical applications in the endodontic treatment.
\end{abstract} (C) 2008 The Electrochemical Society. [DOI: 10.1149/1.2844440] All rights reserved.

Manuscript submitted April 12, 2007; revised manuscript received November 26, 2007. Available electronically March 11, 2008.

The success of endodontic treatment depends on the accurate measurement of root canal length; ${ }^{1-3}$ various approaches, such as tactile sense and radiographic and electronic techniques, have been employed to locate the canal apex of a diseased tooth. The tactile sense is empirical and relatively unreliable. The radiographic technique is also applied in endodontic treatment, but opens to observer interpretation that may result in under-measurement or overmeasurement of canal apex position. ${ }^{4,5}$ Accordingly, the accuracy of these two methods is limited and depends on the experience of the dentist.

The electronic technique for assessing root canal length has attracted considerable attention over the last few decades. Instead of the visual inspection as used in the radiographic technique, it utilizes measurements of the electrical resistance or impedance between the measuring electrode (file), which is inserted into the diseased root canal, and the other large-surface neutral electrode (lip clip), which is placed on the buccal mucosa or held in the patient's hand. Suzuki ${ }^{6}$ measured the electrical resistance between the periodontal ligament and oral mucosa and registered a consistent value of $\sim 6.5 \mathrm{k} \Omega$, independently of the age of the patient or the shape and type of tooth. Sunada ${ }^{7}$ employed this principle and designed a clinical instrument for measuring root canal length. Subsequently, several instruments (called electronic apex locators) based on alternating current with a single frequency or multifrequencies have been developed. ${ }^{8-10}$ These frequency-dependent instruments are based on the electrical principle that the impedance changes substantially when the file approaches the apex of the tooth, in which the root canal is at its narrowest. Although the electronic apex locators can reduce the treatment time and the radiation dose, two main factors, the presence of electrolyte in the root canals and the apical size of the root canal, hamper the precision of the electronic apex locator. ${ }^{11,12}$ The complicated electrical mechanisms associated with the electrical components must be better understood, based on an electrochemical view of the nonfaradaic impedance system, to improve the accuracy of electronic apex locator.

However, most dentistry-related studies have evaluated the accuracy of commercially available instruments in vivo or in vitro. ${ }^{13-17}$ Surprisingly, little fundamental work has been performed on related electrical characteristics and mechanisms. In this study, the completed equivalent circuit model for the complicated electrical mechanism of the endodontic treatment is developed and used to simulate the experimental results. On the basis of the conditions of the electrolyte in the endodontic treatment, the impedance characteristics of

${ }^{\text {z } E-m a i l: ~ s c y e n @ n t u . e d u . t w ; ~ p i n l i n @ n t u . e d u . t w ~}$ a mimic root canal are examined from the perspective of the electrochemical system. Furthermore, new indices numbers $\left(Q\right.$ and $\left.S_{Q}\right)$ are defined to support more precise location of the apex. This investigation will help us to elucidate the complicated mechanisms of electronic endodontic treatment and improve clinical dental applications.

\section{Experimental}

An acrylic test cell with an area of $6.0 \times 6.0 \mathrm{~mm}$ and length of $15.0 \mathrm{~mm}$ shown in Fig. 1a, was used to mimic a human tooth. A cylindrical hollow tubule to mimic the root canal with a $0.2 \mathrm{~mm}$ bottom apex diameter and a $1.4 \mathrm{~mm}$ top diameter was prepared using an acrylic cell under a stereoscopic microscope. Although the electrical characteristics of the acrylic test cell with the cylindrical tubule differ from those of a human tooth, the use of an acrylic tubule to mimic root canals may help eliminate bias in the impedance measurement that results from canal curvature, canal taper, or the latent lateral canals. In addition to the mimic cell, an extracted human tooth with a straight single canal was also prepared by removing crowns to the cementum-enamel junction level and enlarging the corona to a top diameter of $1.0 \mathrm{~mm}$ with an orifice opener. As presented in Fig. 1b, the length and apex diameter of the extracted human tooth were 11 and $0.2 \mathrm{~mm}$, respectively. Following preparation, the extracted human tooth was stored in normal saline solution $(0.9 \% \mathrm{NaCl})$.

As shown in Fig. 1c, electrical impedance measurements of the test cell or the extracted human tooth were made. The test cell or extracted human tooth was mounted onto the polypropylene holder $(20 \mathrm{~mL})$, and then $5 \%$ agar in normal saline solution was gelled within the holder to mimic the periodontal tissue. The bottom surface of the test cell just touched the agar gel surface to prevent the agar from moving into the tubules, yielding erroneous measurements. Then the electrolyte was injected into the tubules using a syringe until they were completely full. The electrolyte used herein was either distilled water or normal saline.

A no. 15 stainless steel file with a length of $18.0 \mathrm{~mm}$ (0.02 taperoff, Profile, Densply Maillefer Co.) and a curved stainless steel lip chip with a large area, which rested in the agar gel, were used as the measuring electrode and neutral electrode, respectively. The electrical impedance between the file and the neutral electrode was measured using an electrochemical impedance measuring system that comprised a potentiostat/galvanostat (AutoLab PGSTAT 30, ECO Chemie) with a frequency response analyzer (FRA) module and FRA 4.9 control software. A small output potential signal from the FRA was sinusoidal with an amplitude of $10 \mathrm{mV}$ based on the open- 
(a)

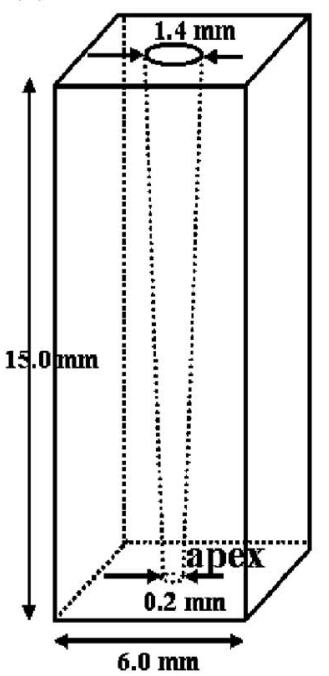

(b)

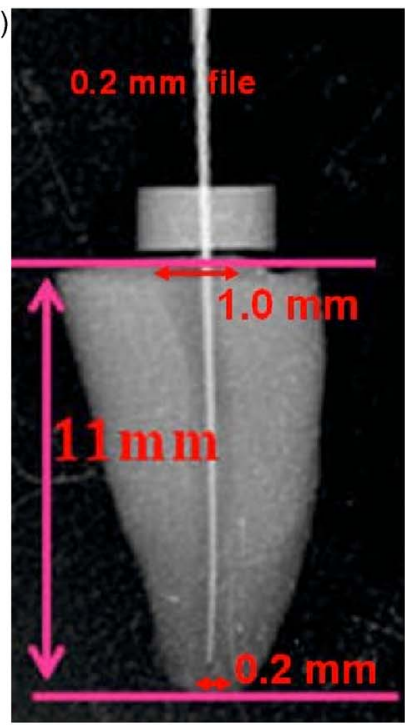

$6.0 \mathrm{~mm}$

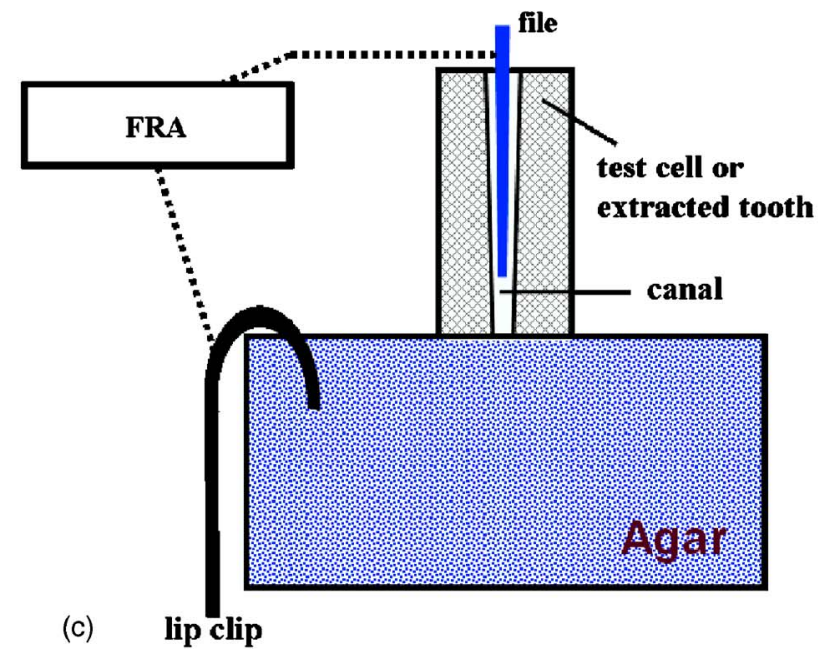

Figure 1. (Color online) Cylindrical hollow tubule with $0.2 \mathrm{~mm}$ apex diameter and $1.4 \mathrm{~mm}$ orifice diameter tunneled through an acrylic block with area of $6.0 \times 6.0 \mathrm{~mm}$ and length of $15.0 \mathrm{~mm}$, (b) extracted human tooth with apex diameter of $\sim 0.2 \mathrm{~mm}$ and length of $11.0 \mathrm{~mm}$, and (c) experimental setup for measuring impedance.

circuit potential and an applied frequency from $30 \mathrm{kHz}$ to $10 \mathrm{~Hz}$. Electrochemical impedance spectroscopy was performed using the characteristics of the mimic system.

The measuring file was steadily inserted along the canal using a micrometer screw gauge until it was $>2 \mathrm{~mm}(+2.0 \mathrm{~mm})$ from the apex. The pitch of the screw was $0.5 \mathrm{~mm}$, and the lowest value was $0.01 \mathrm{~mm}$. The impedance of each filling electrolyte was measured as the file was placed at $x=-3.0,-1.0,-0.5,-0.3,-0.1,0$, $+0.1,+0.3,+0.5,+1.0$, and $+2.0 \mathrm{~mm}$ from the apex (where negative and positive signs represent the underapex and overapex, respectively).

\section{Tooth Impedance from the Perspective} of an Electrochemical System

The behavior of typical electrical components, such as resistors, capacitors, and inductors, is well documented. ${ }^{18}$ Meredith and Gulabivala ${ }^{19}$ measured the in vivo electrical impedance characteristics of a root canal using an LCR analyzer and proposed a simple equivalent circuit to model the impedance between the root canal

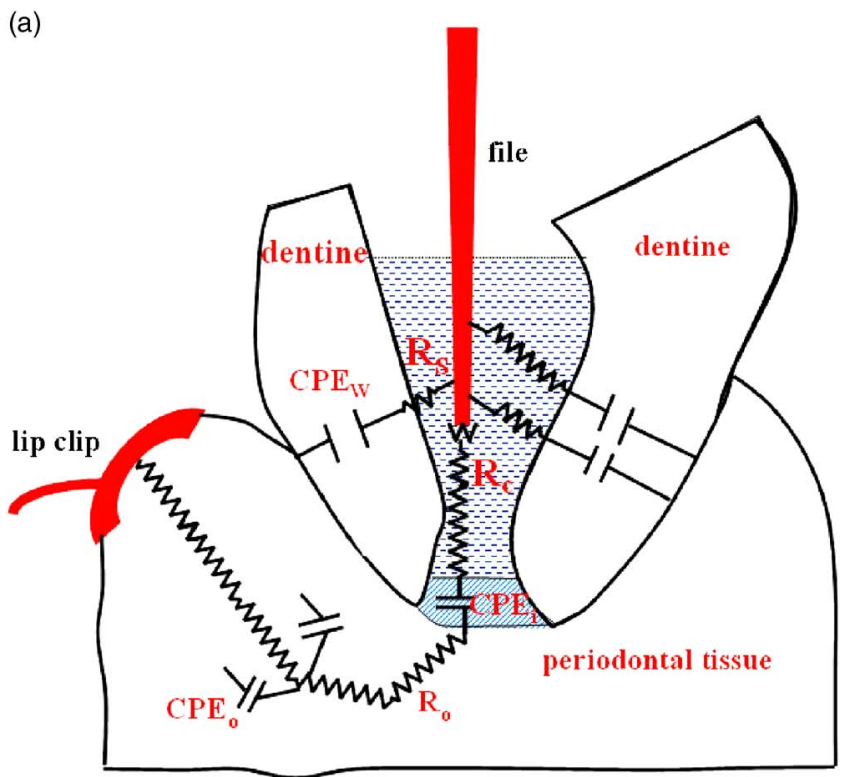

(b)
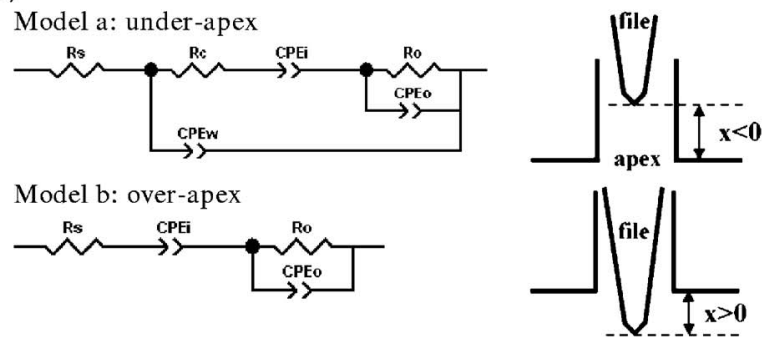

Figure 2. (Color online) (a) Schematic proposed circuit model: $R_{\mathrm{S}}$ is the resistance of the electrode surface and the lateral-side solution resistance of the file dipped into the canal. $R_{\mathrm{c}}$ and $R_{\mathrm{o}}$ are the resistances of the solution from the file tip to the apex and of the periodontal tissue, respectively. $\mathrm{CPE}_{\mathrm{w}}$, $\mathrm{CPE}_{\mathrm{i}}$, and $\mathrm{CPE}_{\mathrm{o}}$ represent the capacitative reactances of the walls of dentin plus stray capacitative reactance, the solution-agar interface and the medium outside canal, respectively. (b) Corresponding equivalent circuit models of the tubule-agar system; model $\mathrm{a}$ and $\mathrm{b}$ are employed to simulate the experimental results of the file located at the under-apex $(x \leqq 0)$ and over-apex $(x>0)$, respectively.

and the buccal mucosa. The series and parallel resistive components in their proposed equivalent circuit were suggested to be associated with the surface effect and bulk phenomenon, respectively. However, no further experimental results on this equivalent circuit are available.

From the perspective of the electrochemical system, the impedance response between the human tooth and the periodontal tissue can be modeled as a combination of passive electrical components. As displayed in Fig. 2a, the narrow root canals of the human tooth, which contain such conductive materials as nerves, soft tissue, electrolytic and medicinal fluid, are surrounded by dentine with high inherent resistivity. Accordingly, the electric behavior of a root canal resembles that of a resistor and depends on the dimensions and the inherent resistivity of the conductive fluid. However, the electrical behavior of dentine is more like that of a capacitor and depends on the dimensions and the dielectric constant for alternating current. At the apex of the root canal is a small hole that is connected to the periodontal tissue, which serves as an electric conductor. The interface between the electrolyte and the periodontal tissue behaves like an interfacial capacitor.

The constant phase element (CPE) is frequently adopted in a model of most real electrochemical systems, in place of the ideal 
capacitor, to compensate for nonhomogeneity and nonideality of the system, such as associated with a rough or porous surface. ${ }^{20,21}$ The $\mathrm{CPE}$ is given by

$$
Z=\frac{1}{Q(i \omega)^{n}}
$$

where $Q$ is expressed in ohms per second $(Q$ is the ideal perfect capacitance $C$ when $n=1$ ). As $n$ approaches 1 and 0 , the CPE behaves more like an ideal capacitor and ideal resistor, respectively. The value of $n$ for a component in an electrochemical system is typically between 0.5 and unity.

Križaj ${ }^{22}$ presented a Fricke model (a resistor and a CPE element in series) rather than a resistance-capacitance model (a resistor and a capacitor in series) to elucidate the equivalent circuit; the file was located over the apical end point. Their results also indicated that the equivalent circuit model became more complex when the file was inside the root canal because part of the current that flows through the dentin layer should be taken into account by adding a capacitor and a resistor. However, their experimental data do not match the fitted results very closely. Few studies have addressed the equivalent circuit of electrically endodontic treatment, and the related complicated electrical mechanisms remain unclear and the relevant electrical components require more investigation.

\section{Results and Discussion}

Figure 2 depicts an equivalent circuit model of the measuring system between the file and the lip chip electrode. $R_{\mathrm{S}}$ denotes the resistance of the electrode surface and the lateral-side solution resistance of the file that was dipped into the canal. $R_{\mathrm{c}}$ and $R_{\mathrm{o}}$ represent the resistance of the solution from the file tip to the apex and the periodontal tissue, respectively. $\mathrm{CPE}_{\mathrm{w}}, \mathrm{CPE}_{\mathrm{i}}$, and $\mathrm{CPE}_{\mathrm{o}}$ represent the capacitative reactances of the walls of the dentin plus stray capacitative reactances, the solution-agar interface, and the medium outside the canal, respectively. $R_{\mathrm{s}}$ depends on the lateral geometric dimension of the electrode that was dipped in the canal and the inherent conductivity of the solution ( $\kappa)$. The side resistance of the $R_{\mathrm{S}}$ is given by

$$
R_{\mathrm{s}} \approx \frac{d}{\mathrm{\kappa} P}
$$

where $d$ and $P$ represent the effective distance from the electrode to the dentin wall and the surface area of the electrode dipped in the canal, respectively. The value of $R_{\mathrm{c}}$ depends on the geometric dimensions from the file tip to the apex and the inherent conductivity of the solution, $\kappa_{\mathrm{s}}$, in the canal. Hence, $R_{\mathrm{c}}$ is

$$
R_{\mathrm{c}} \approx \frac{L}{\kappa_{\mathrm{s}} A}
$$

where $L$ and $A$ represent the efficient distance from the file tip to the apex and the cross-sectional area of the apex, respectively. The resistance $R_{0}$ could be treated as the expansive resistance that steadies the current flow from a disk electrode with radius $r_{0}$ into a semiinfinite medium; this is also the bulk resistance of the periodontal tissue. The value of $R_{0}$ depends on the diameter of the apex diameter, $D$, and the bulk conductivity of the periodontal tissue, $\kappa_{0}$, in the canal. Therefore, $R_{0}$ can be expressed as

$$
R_{0} \approx \frac{1}{\mathrm{\kappa}_{0} D}
$$

The CPE, which replaced the ideal perfect capacitance, $C$, was used in the simulation of the experimental results to elucidate the characteristics of the real electrode surface. The total impedance, $Z_{\mathrm{t}}$, of the measuring system is given by

$$
\begin{gathered}
Z_{\mathrm{t}}=R_{\mathrm{s}}+Z_{3} \\
\frac{1}{Z_{3}}=\frac{1}{Z_{2}}+Q_{\mathrm{w}}(i \omega)^{n_{\mathrm{w}}}
\end{gathered}
$$

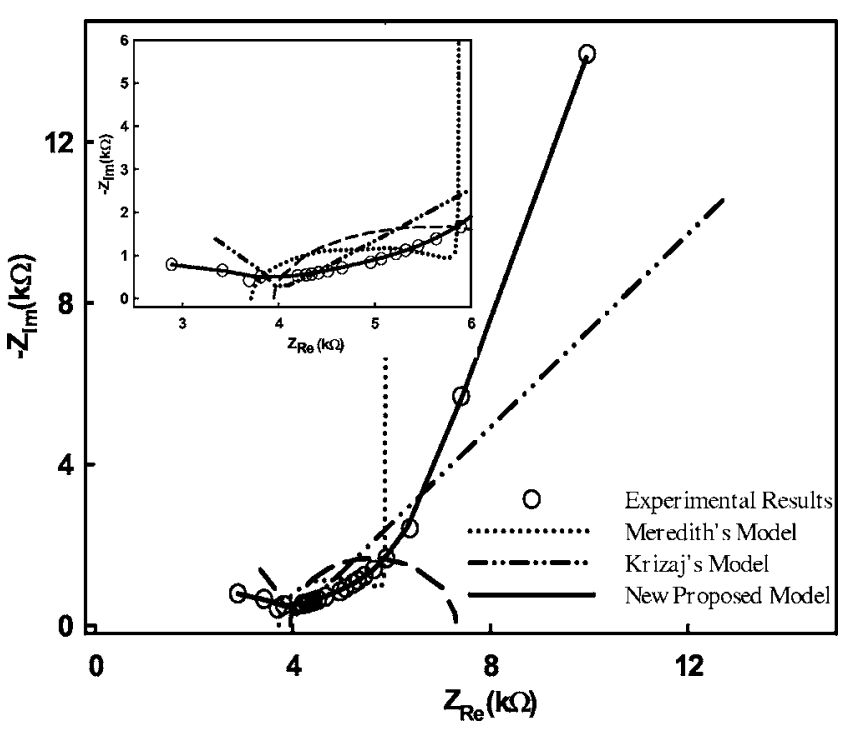

Figure 3. Impedance response of measurement system based on extracted tooth filled with normal saline, with measuring file positioned $1 \mathrm{~mm}$ under the apex.

$$
\begin{gathered}
Z_{2}=R_{\mathrm{c}}+\frac{1}{Q_{\mathrm{i}}(i \omega)^{n_{\mathrm{i}}}}+Z_{1} \\
\frac{1}{Z_{1}}=\frac{1}{R_{0}}+Q_{0}(i \omega)^{n_{0}}
\end{gathered}
$$

Figure 3 plots the impedance response of the extracted tooth that is filled with normal saline when the measuring file is positioned $1 \mathrm{~mm}$ under the apex. Three equivalent circuits, Meredith's, ${ }^{19}$ Križaj's, ${ }^{22}$ and our circuit, were applied to simulate the experimental impedance response of the measuring system using the nonlinear least-squares algorithm with the best agreement. The experimental results differed substantially from those obtained using Meredith's model. The results simulated using Križaj's model differed much less, but the simulation clearly did not yield the experimental impedance response with complete accuracy. Our model, presented in Fig. 3, was employed to simulate the experimental impedance response of the measuring system well with the best agreement (error $\leqq 0.1 \%)$. It also provided significant physical information regarding the complicated electrical characteristics of the human tooth system.

Figures 4 and 5 plot the impedance responses of the extracted tooth and acrylic tubule for the canal filled with normal saline, respectively. In Fig. 4 and 5, the small quarter-circles appear in the region of high frequency and the relatively large curve is in the region of low frequency, when the file was in the under-apex location. The size of the quarter-circle declines closer to the apex. When the file reaches the apex, the quarter-circle is invisible and only the curves remain. Accordingly, the quarter-circular impedance responses in the region of high frequency are related to the electrical characteristics of the interior of the canal, including $R_{\mathrm{S}}, R_{\mathrm{c}}$, and $\mathrm{CPE}_{\mathrm{w}}$.

From Table I, $R_{\mathrm{S}}$ and $R_{\mathrm{c}}$ decrease as the file approaches the apex because the electrode surface dipped-area $(P)$ increases and the relative distance from the file tip to apex $(L)$ decreases. The impedance responses of the relatively large curves at low frequency are associated with the characteristics outside the canal, including the parallel combination of the resistance of the medium outside the canal, $R_{\mathrm{o}}$, and the capacitative reactance of the medium outside the canal, $\mathrm{CPE}_{\mathrm{o}}$, in series with the capacitative reactance of the interface $\mathrm{CPE}_{\mathrm{i}}$. Large variations of the $R_{\mathrm{o}}$ and $Q_{\mathrm{i}}$ values occur when the file is at the critical position of the apex $(x=0)$ due to the change of the geometry and the electrolyte-agar interface. 


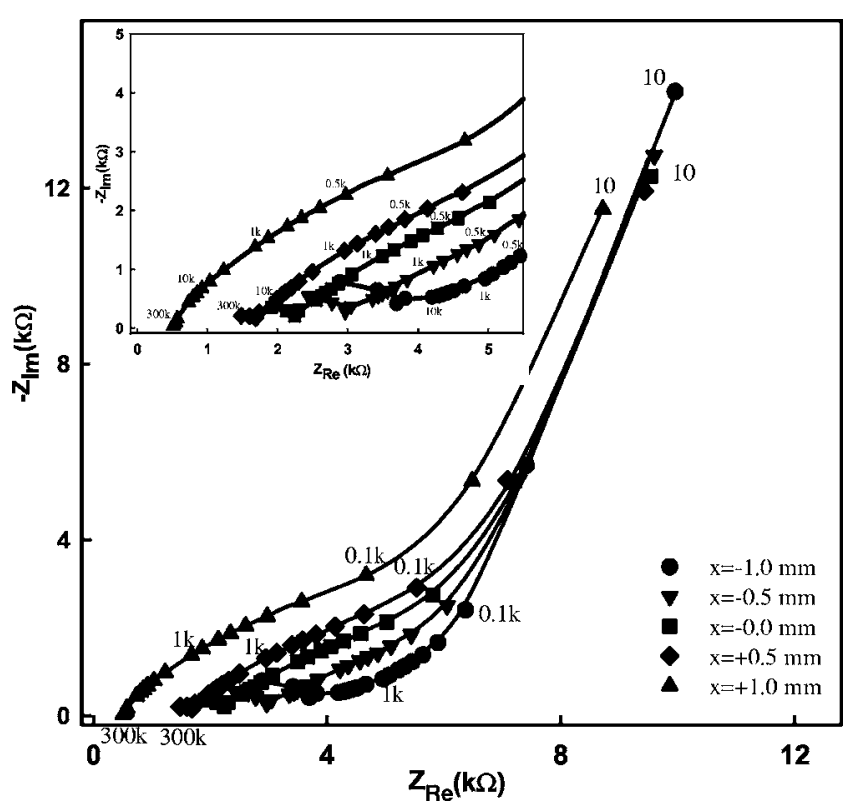

Figure 4. Impedance response of measured system with extracted tooth filled with normal saline. Symbols and lines represent experimental data and results simulating using equivalent circuits, respectively. Numbers around symbols represent the measuring frequencies.

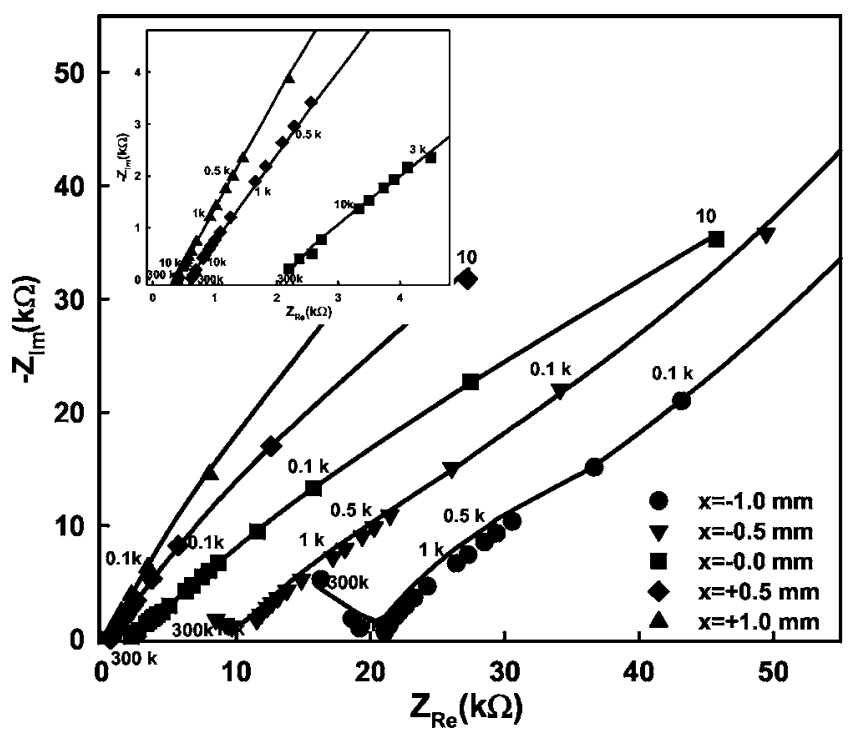

Figure 5. Impedance response of measurement system with acrylic tubule filled with normal saline. Symbols and lines represent experimental data and results simulated using equivalent circuits, respectively. Numbers around symbols represent the measuring frequencies.

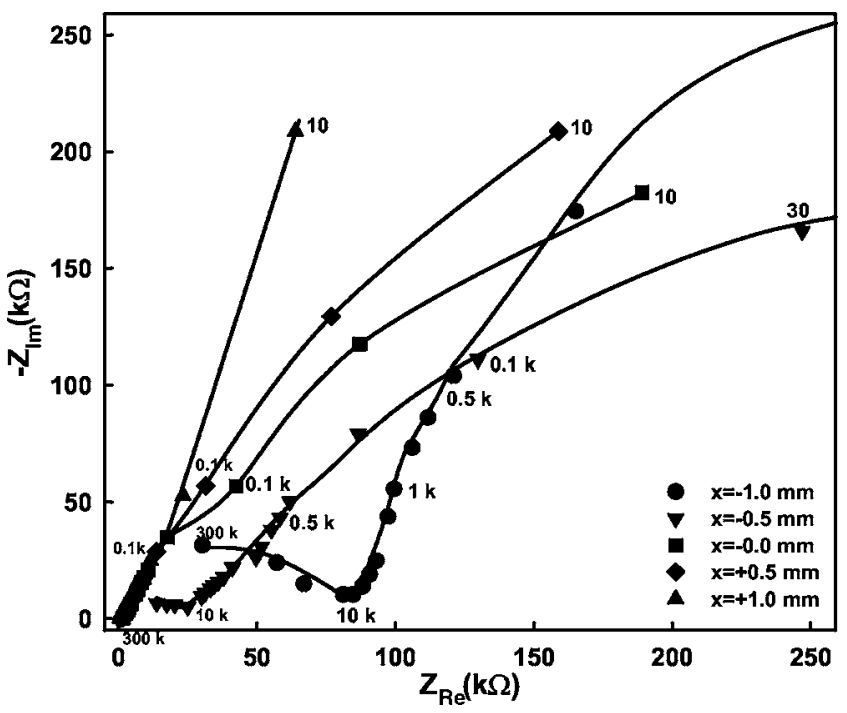

Figure 6. Impedance response of measurement system with acrylic tubule filled with distilled water. Symbols and lines represent the experimental data and the results simulated based on the equivalent circuit, respectively. Numbers around symbols represent the measuring frequencies.

Figure 6 shows the impedance response of the tubule that was filled with distilled water. These experimental impedance results were similar to those of the canal, which were filled with normal saline, revealing that the electrical mechanism and characteristics of all electrolytes were very much alike. However, the impedances of the tubule filled with distilled water were much larger than those filled with normal saline because the conductivity of distilled water is low. Accordingly, the value of the $R_{\mathrm{c}}$ significantly decreases as the file approaches the apex.

Figures 7 and 8 plot the impedance magnitude $(|Z|)$ of the tubule filled with normal saline and distilled water, respectively. In Fig. 7, the magnitude of the impedance in the under-apex and the over-apex regions declined approximately linearly as the file approached the apex at each frequency. As the measuring frequency increased, the impedance magnitude declined substantially. The variations of impedances with any two frequencies gradually increased as the file approached the apex. Accordingly, the ratio of the impedance values at the two frequencies $(|Z|$ at low frequency divided by $|Z|$ at high frequency) declines as the file approaches the apex. The ratio of the capacitive reactance to the total impedance gradually increases as the file approaches the apex. These characteristics demonstrate that the impedance-based measuring system with a single frequency cannot easily yield the apex location accurately under endodontic treatment. Figure 8 also indicates that the experimental results for the tubule filled with distilled water were similar to those for the canal

Table I. Simulated values of measured system with acrylic tubule filled with normal saline and distilled water.

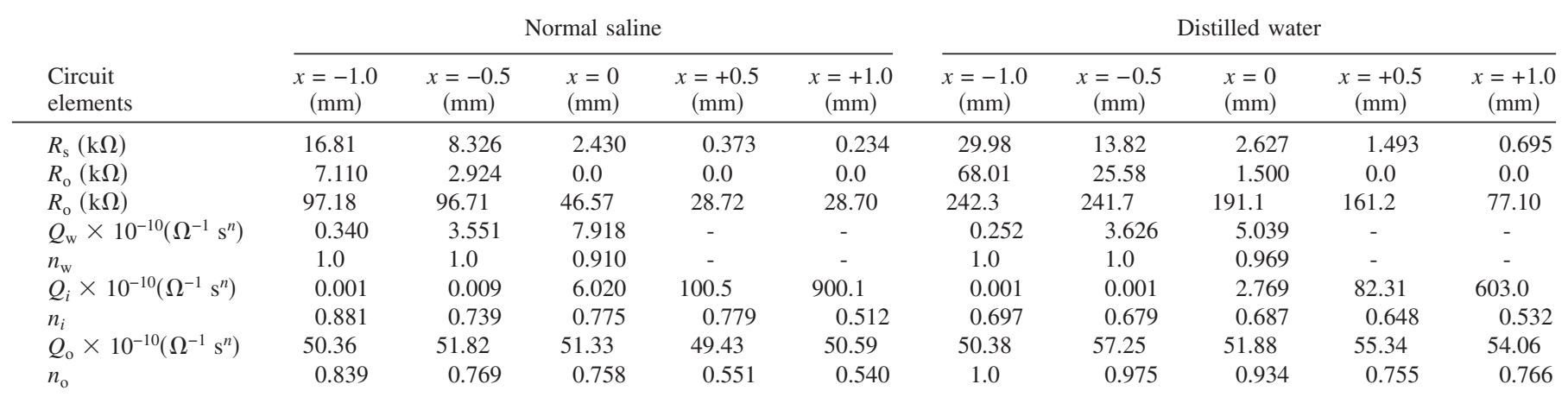




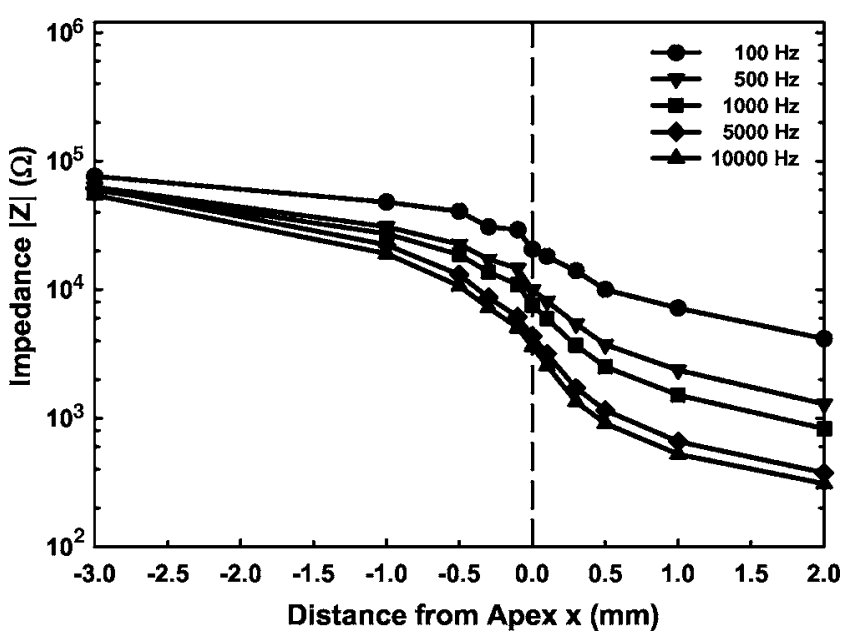

Figure 7. Impedance $(|Z|)$ at various distances from apex $(x)$ for tubule filled with normal saline.

filled with normal saline. However, the magnitude of the impedance in the under-apex fell markedly, compared to that for the canal that was filled with normal saline. This result reveals the fact that the impedances of the canal filled with electrolytes with lower conductivity depend more strongly on the distance from the apex because the effective resistance of the electrolytic solution in the canal is larger.

Because the variation in the impedance with frequency slowly increases as the file approaches the apex, as plotted in Fig. 7 and 8, the modified quotient of the impedance, $Q$, was introduced to indicate the variation in impedance between high frequency and low frequency. $Q$ is defined by

$$
Q=\frac{\Delta|Z|}{|Z|_{\text {avg. }}}=\frac{|Z|_{\text {low }}-|Z|_{\text {high }}}{\frac{\left(|Z|_{\text {low }}+|Z|_{\text {high }}\right)}{2}}
$$

where $|Z|_{\text {low }}$ and $|Z|_{\text {high }}$ are the $|Z|$ values at low and high frequencies, respectively. The capacitive reactance is inversely proportional to the measuring frequency, and the resistance is independent of it. Therefore, $Q$ approaches 0 and 2 as the resistance and the reactance dominate, respectively. Figure 9 shows the modified quotient of the impedance (dashed line) at the various locations for two pairs of frequencies $\left(f=10 \times 10^{3}, 100 \mathrm{~Hz}\right.$ and $\left.5 \times 10^{3}, 500 \mathrm{~Hz}\right) . Q$ re-

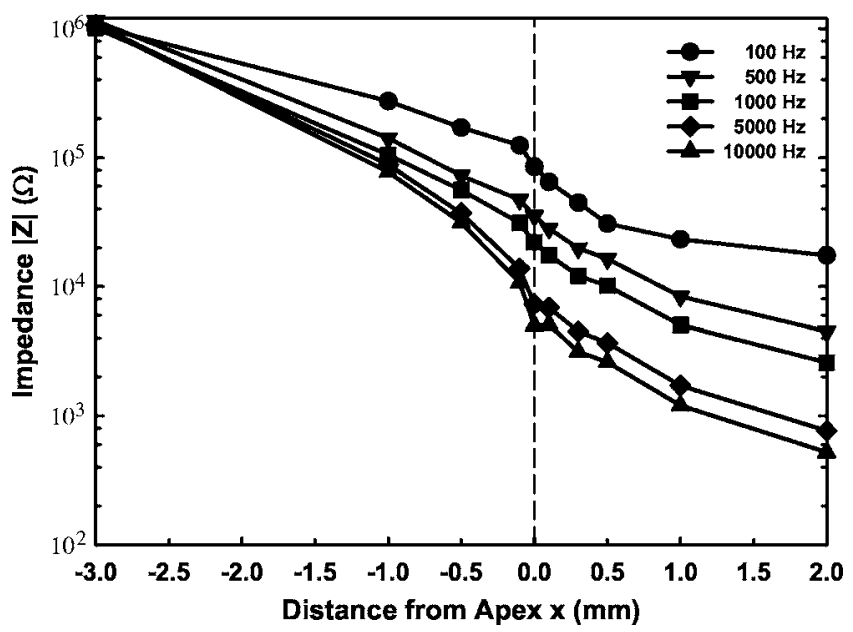

Figure 8. Impedance $(|Z|)$ at various distances from apex $(x)$ for tubule filled with distilled water.

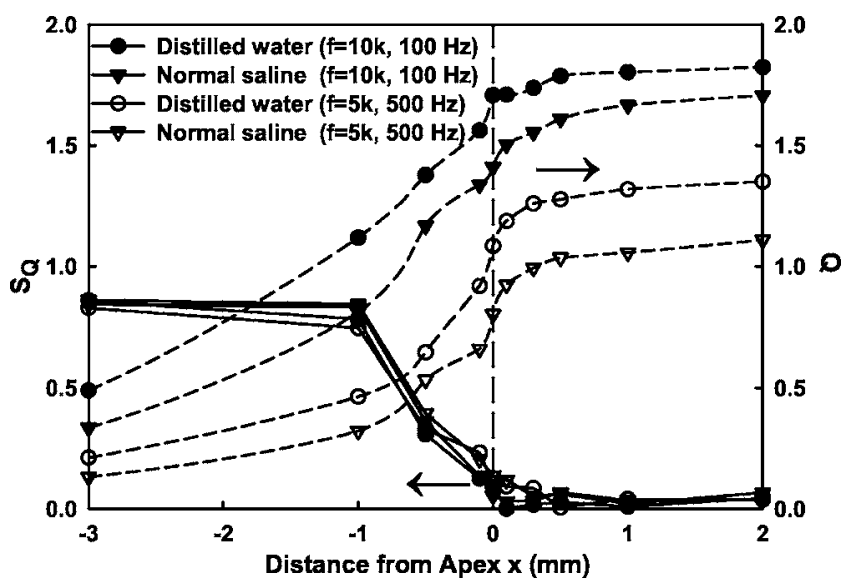

Figure 9. Dependence of modified quotient of impedance ( $Q$, dashed line) and impedance-quotient variation ratio $\left(S_{Q}\right.$, solid line) for various electrolytes.

vealed different impedance behaviors in the under-apex and overapex regions, respectively. The impedance characteristics shifted from resistance dominated to reactance dominated as the file approached the apex. The modified quotient of the impedance in the under-apex region clearly depended on the distance from apex $x$ and the electrolytic conductivity $\kappa_{\mathrm{s}}$. However, the dependence of $Q$ on the distance from the apex in the over-apex region was much weaker than that in the under-apex region because the impedance response in the over-apex region is that of bulk agar gel, which corresponds to the impedance response of the periodontal tissue. To determine the apex for any electrolyte, the variation ratio of the impedance quotient, $S_{Q}$, is defined as

$$
S_{Q}=\frac{\Delta|Q|}{|Q|_{\text {avg. }}}=\frac{|Q|_{x=x_{2}}-|Q|_{x=x_{1}}}{\frac{\left(|Q|_{x=x_{2}}+|Q|_{x=x_{1}}\right)}{2}}
$$

where $x_{1}$ is the measurement location previous to $x_{2}$. Figure 9 plots the variation ratio of the impedance-quotient (solid line) at the various positions. Figure 9 also demonstrates that the variation ratio of the impedance quotient declines as the files approach the apex; the values are much smaller in the over-apex region. The impedancequotient variation ratio is below 0.15 at the apex and in the overapex region for all electrolytes. Moreover, the variation ratio of the impedance quotient is almost independent of the conductivity of the electrolyte and the selected measuring frequency. Therefore, the impedance-quotient variation ratio, $S_{Q}$, is an index, which can be used to eliminate the effect of the electrolytic conductivity to locate the root canal. When $S_{Q}$ first falls below 0.15 , the file tip can be regarded as being at the apex.

\section{Conclusions}

Although the electrical mechanisms associated with in vivo human tooth root canals may be much more complicated, the fullspectrum experiments that involved a tubule or an extracted tooth to replicate root canals help to provide relevant information. In this work, the electrical impedance characteristics of the tubules filled with either normal saline or distilled water were investigated over the frequency spectrum. An appropriate equivalent circuit model was proposed to measure the electric impedance of canals of various lengths in the dental application. Because the precise location of a root canal apex is one of the most important steps in endodontic treatment, the modified quotient of the impedance and the variation ratio of the impedance quotient can be used as significant indices for the impedance characteristics and location. The impedance-quotient variation ratios are $<0.15$ at the apex and in the over-apex region, regardless of electrolyte. Additionally, the variation ratio of the im- 
pedance quotient can be applied as an index to locate the apex, where the effect of conductivity of any electrolyte is eliminated.

National Taiwan University assisted in meeting the publication costs of this article.

\section{References}

1. A. Katz, A. Tamse, and A. Y. Kaufman, J. Oral Surgery, Oral Medicine, Oral Pathology, Radiol. Endodont., 72, 238 (1991).

2. D. Ricucci, Int. Endod. J., 31, 384 (1998).

3. A. Y. Kaufman, S. Keila, and M. Yoshpe, Int. Endod. J., 35186 (2002).

4. A. Olson, A. Goerig, R. Cavataio, and J. Luciano, Int. Endod. J., 24, 28 (1991)

5. M. A. Martinez-Lozano, L. Forner-Navarro, J. L. Sánchez-Corteś, and C. LlenaPuy, Int. Endod. J., 34, 371 (2001).

6. K. Suzuki, Japanese Journal of Stomatology, 16, 411 (1942)

7. I. Sunada, J. Dent. Res., 41, 375 (1962).

8. N. Inoue and D. H. Skinner, J. Endod., 11, 421 (1985).
9. T. Saito and Y. Yamashita, Dent. Jpn. (Tokyo), 27, 65 (1990).

10. C. Kobayashi and H. Suda, J. Endod., 20, 111 (1994).

11. Y. N. Wu, J. N. Shi, L. Z. Huang, and Y. Y. Xu, Int. Endod. J., 25, 88 (1992).

12. L. Z. Huang, J. Endod., 13, 60 (1987).

13. J. Jenkins, W. Walker, W. Schindler, and C. Flores, J. Endod., 27, 209 (2001).

14. F. Goldberg, A. C. De Silvio, S. Manfré, and N. Nastri, J. Endod., 28, 461 (2002).

15. A. C. Tinaz, L. S. Sivimli, G. Görgül, and E. G. Türköz, J. Endod., 28, 160 (2002)

16. A. R. Welk, J. C. Baumgartner, and J. G. Marshall, J. Endod., 29, 497 (2003).

17. W. Fan, B. Fan, J. L. Gutmann, Z. Bian, and M. W. Fan, J. Endod., 39, 127 (2006).

18. A. J. Bard and L. R. Faulkner, Electrochemical Methods: Fundamentals and Applications, 2nd Ed., chap. 10, Wiley, New York (2001).

19. N. Meredith and K. Gulabivala, Endod. Dent. Traumatol., 13, 126 (1997).

20. E. Barsoukov and J. R. Macdonald, Impedance Spectroscopy: Theory, Experiment, and Applications, 2nd Ed., p. 34, Wiley, New York (2005).

21. V. A. Alves, L. A. da Silva, and J. F. C. Boodts, Electrochim. Acta, 44, 1525 (1998).

22. D. Križaj, J. Jan, and V. Valenčič, Bioelectromagnetics (N.Y.), 25, 185 (2004) 